\title{
Algebraic Approach to Baryon Structure
}

\author{
A. Leviatan \\ Racah Institute of Physics, The Hebrew University, \\ Jerusalem 91904, Israel \\ R. Bijker \\ Instituto de Ciencias Nucleares, U.N.A.M., A.P. 70-543, \\ 04510 México, D.F., México
}

\begin{abstract}
We present an algebraic approach to the internal structure of baryons in terms of three constituents. We investigate a collective model in which the nucleon is regarded as a rotating and vibrating oblate top with a prescribed distribution of charges and magnetization. We contrast the collective and single-particle descriptions of baryons and compare the predictions of the model with existing data on masses, electromagnetic elastic and transition form factors and strong decays widths.
\end{abstract}

Invited talk at 'Symmetries in Science IX',

Bregenz, Austria, August 6-10, 1996 


\section{Introduction}

In the usual description of baryons in terms of three constituents, the wave-function is a product of a space part and an internal spin-flavor-color part. Algebraic methods have been used extensively in the past to describe the internal part in terms of the symmetry groups $S U_{s f}(6) \otimes S U_{c}(3)$ [1, 2]. The difference between models lies in different assumptions on the spatial dynamics of the three constituents. Quark potential models in nonrelativistic [3] or relativized [4] forms emphasize the single-particle aspects of quark dynamics for which only a few low-lying configurations in the confining potential contribute significantly to the eigenstates of the Hamiltonian. Excited baryons in this description correspond to single-particle excitations of levels in the confining potential. On the other hand, flux-tube models, soliton models as well as some regularities in the observed spectra (e.g. linear Regge trajectories, parity doubling) hint that an alternative, collective type of dynamics may play a role in the structure of baryons.

The aim of the present contribution is to report on an algebraic framework [5] which encompasses both the single-particle and collective scenarios. The formalism is based on a $U(7)$ spectrum generating algebra whose bosonic realization and geometry were discussed at length in a previous contribution to these proceedings [6]. The algebraic formulation allows us to study a large class of models, all with the same spin-flavor-color structure, but different types of spatial dynamics. Among the models is the familiar harmonic oscillator quark model taken as a proto-type for single-particle dynamics, and a collective model in which the baryon resonances are interpreted as rotations and vibrations of an oblatetop shaped string with a distribution of charge magnetic moments. In what follows we report on an application of the model to the mass spectrum of nonstrange baryons, derive expressions of form factors relevant for electromagnetic and strong couplings [5]. We examine the effect of spin-flavor breaking and stretching on the electromagnetic helicity amplitudes [7] and calculate strong decay widths [8]. The predictions of the collectivemodel are compared with those of the single-particle description and with experimental data on these observables. The reader is encouraged to consult first [6] and to find more 
details in [5, 7, 8, 9].

\section{Mass Spectrum}

We consider the baryon as an object composed of three identical constituents carrying internal quantum numbers: flavor $=$ triplet $=u, d, s$; spin $=$ doublet $=1 / 2$; and color $=$ triplet. The geometric arrangement of this three-body system can be phrased in terms of two relative Jacobi vectors. It is possible [5, 6] to cast the dynamics of these spatial degrees of freedom in algebraic form, in terms of a $U(7)$ spectrum generating algebra. The full algebraic structure is obtained by combining the geometric part, with the usual spin-flavor-color part, resulting in total wave functions which are representations of $U(7) \otimes$ $S U_{s f}(6) \otimes S U_{c}(3)$

$$
\left|{ }^{2 S+1} \operatorname{dim}\left\{S U_{f}(3)\right\}_{J}\left[\operatorname{dim}\left\{S U_{s f}(6)\right\}, L^{P}\right]_{\left(v_{1}, v_{2}\right) ; K}\right\rangle
$$

The classification under $S U_{s f}(6)$ and its flavor-spin subgroups $S U_{f}(3) \otimes S U_{S}(2)$, are denoted by the dimension of the corresponding irreducible representations. We consider a collective model in which nonstrange baryons are interpreted as excitations of the string configuration in Fig. 1. In this case the spatial part in Eq. (2.1) consists of an oblatetop wave function [6] characterized by the labels: $\left(v_{1}, v_{2}\right) ; K, L_{t}^{P}$, where $\left(v_{1}, v_{2}\right)$ denotes the vibrations (stretching and bending) of the string; $K$ denotes the projection of the rotational angular momentum $L$ on the body-fixed symmetry-axis; $P$ the parity and $t$ the $S_{3}$ symmetry type of the state under permutations. The permutation symmetry of the spatial part must be the same as that of the spin-flavor part to ensure antisymmetry of the full space-spin-flavor-color wave-function $\left(S U_{s f}(6)\right.$ species: [56] symmetric, [20] antisymmetric, [70] mixed symmetry). The spin $S$ and $L$ are coupled to total angular momentum $J$. In this notation the nucleon $(S=1 / 2$, flavor octet) and delta $(S=3 / 2$ flavor decuplet) ground state wave functions are given by

$$
\left|{ }^{2} 8_{1 / 2}\left[56,0^{+}\right]_{(0,0) ; 0}\right\rangle \quad \text { and } \quad\left|{ }^{4} 10_{3 / 2}\left[56,0^{+}\right]_{(0,0) ; 0}\right\rangle \text {. }
$$


For harmonic oscillator dynamics, the spin-flavor part in is the same, while in the spatial part the $\left(v_{1}, v_{2}\right) ; K$ labels are replaced by the harmonic oscillator quantum number $n$.

An $S_{3}$-invariant operator can now be constructed along the lines outlined in [6], and used to describe properties of nucleon and delta resonances. In the collective model the resulting mass spectrum exhibits rotational states $\left(L_{t}^{P}, K\right)$ arranged in bands built on top of each vibration $\left(v_{1}, v_{2}\right)$. For a large model space the spectrum follows closely a mass formula of the form

$$
M^{2}=M_{0}^{2}+M_{\mathrm{vib}}^{2}\left(\lambda_{1}, \lambda_{2}\right)+M_{\mathrm{rot}}^{2}(\alpha)+M_{\mathrm{spin}-\text { flavor }}^{2}(a, b, c) .
$$

The spatial $U(7)$ contribution to the mass consists of a vibrational part $\left(M_{\mathrm{vib}}^{2}=\lambda_{1} v_{1}+\right.$ $\left.\lambda_{2} v_{2}\right)$ and a rotational part $\left(M_{\text {rot }}^{2}=\alpha L\right)$. The spin-flavor contribution is expressed in a Gürsey Radicati form [2] associated with the chain $S U_{s f}(6) \supset S U_{f}(3) \otimes S U_{s}(2)$. The resulting fit (r.m.s deviation of $39 \mathrm{MeV}$ ) for $3 *$ and $4 *$ nucleon and delta resonances is shown in Fig. 2, along with a comparison to the non-relativistic [3] and relativized [4] quark models. As can be seen, the quality of the fits are comparable although the underlying dynamics and corresponding wave functions are different. A typical oblate-top collective wave-function for the ground state is shown in Fig. 3, expanded in an harmonic oscillator basis. It exhibits strong mixing of many oscillator shells and reflects a correlated motion of the constituents. This significant spread should be compared to the structure of the ground state in the quark potential model [10] where $81 \%$ of the wave function is a pure $n=0$ configuration and the $19 \%$ admixture of $n=2$ components is induced by the hyperfine interaction. The above analysis of the spectrum in the nonstrange sector, shows that masses alone are not sufficient to distinguish between single-particle and collective forms of dynamics. To do so require an examination of other observables (e.g. electromagnetic and strong couplings) which are more sensitive to the structure of wave-functions. 


\section{Collective Form Factors}

To consider decay processes of baryon resonances we need two ingredients: (i) the wave function of the initial and final states and (ii) the form of the transition operator. It is assumed that the operators inducing the electromagnetic (strong) transitions involve an absorption or emission of a photon (elementary meson) from a single constituent. In such circumstances, the couplings discussed below can be expressed in terms of the operators

$$
\begin{aligned}
\hat{U} & =\mathrm{e}^{-i k \sqrt{\frac{2}{3}} \lambda_{z}} \\
\hat{T}_{m} & =\frac{i m_{3} k_{0}}{2}\left(\sqrt{\frac{2}{3}} \lambda_{m} \mathrm{e}^{-i k \sqrt{\frac{2}{3}} \lambda_{z}}+\mathrm{e}^{-i k \sqrt{\frac{2}{3}} \lambda_{z}} \sqrt{\frac{2}{3}} \lambda_{m}\right)
\end{aligned}
$$

where $\lambda_{m}(m=0, \pm)$ are Jacobi coordinates and $\left(k_{0}, \vec{k}\right)$ is the four-momentum of the absorbed quanta. The form factors of interest are proportional to the matrix elements of these operators. In the algebraic approach, these operators are first mapped onto the $U(7)$ algebra and their matrix elements are evaluated in the wave-functions of Eq. (2.1). The algebraic images of the operators in Eq. (3.1) and the calculation of matrix elements were presented in [6]. Table I in [6] shows the resulting elementary form factors corresponding to the case in which the charge and magnetization are concentrated at the end points of the string of Fig. 1. Different types of collective models are specified by a distribution of the charge, magnetic moment, etc., along the string. For the present analysis we use the (normalized) distribution

$$
g(\beta)=\beta^{2} \mathrm{e}^{-\beta / a} / 2 a^{3}
$$

where $\beta$ is a radial coordinate and $a$ is a scale parameter. The collective form factors are obtained by folding the matrix elements of $\hat{U}$ and $\hat{T}_{m}$ with this probability distribution

$$
\begin{aligned}
\mathcal{F}(k) & =\int \mathrm{d} \beta g(\beta)\left\langle\psi_{f}|\hat{U}| \psi_{i}\right\rangle, \\
\mathcal{G}_{m}(k) & =\int \mathrm{d} \beta g(\beta)\left\langle\psi_{f}\left|\hat{T}_{m}\right| \psi_{i}\right\rangle .
\end{aligned}
$$

Here $\psi$ denotes the spatial part of the baryon wave function. The ansatz of Eq. (3.2) for the probability distribution is made to obtain the dipole form for the elastic form 
factor. With the same distribution we can now derive closed expressions for inelastic form factors connecting other final states. A sample of collective transition form factors of the distributed string are given in Table [I, which also lists the corresponding harmonic oscillator form factors. The single-particle and collective form factors differ in their $k$ dependence. In particular, all collective form factors drop as powers of $k$. This property is well-known experimentally and is in contrast with harmonic oscillator quark models in which all form factors which fall off exponentially.

\section{Electromagnetic Couplings}

In electromagnetic processes such as photo- and electroproduction we encounter both elastic transitions involving diagonal matrix elements and inelastic transitions involving non-diagonal matrix elements of the transition operator. We consider below the corresponding observables (elastic electric and magnetic form factors of the nucleon and helicity amplitudes) which can be measured.

\subsection{Elastic Form Factors of the Nucleon}

Taking into account the overall symmetry of the nucleon wave function, the elastic electric $(E)$ and magnetic $(M)$ collective form factors are given by

$$
\begin{aligned}
G_{E}^{N} & =3 \int \mathrm{d} \beta g(\beta)\left\langle\Psi ; M_{J}=1 / 2\left|e_{3} \hat{U}\right| \Psi ; M_{J}=1 / 2\right\rangle \\
G_{M}^{N} & =3 \int \mathrm{d} \beta g(\beta)\left\langle\Psi ; M_{J}=1 / 2\left|\mu_{3} e_{3} \sigma_{3, z} \hat{U}\right| \Psi ; M_{J}=1 / 2\right\rangle
\end{aligned}
$$

where $\Psi$ denotes the nucleon wave function ${ }^{2} 8_{1 / 2}^{N}\left[56,0^{+}\right]_{(0,0) ; 0}$ with $N=p(n)$ for proton (neutron). Further $e_{3}, \mu_{3}=e g_{3} / 2 m_{3}, m_{3}, g_{3}, s_{3}=\sigma_{3} / 2$ are the charge (in units of $e$ : $\left.e_{u}=2 / 3, e_{d}=-1 / 3\right)$, scale magnetic moment, mass, $g$-factor and spin, respectively, of

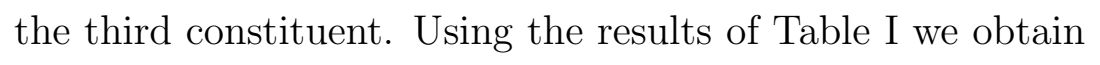

$$
G_{E}^{p}=\frac{1}{\left(1+k^{2} a^{2}\right)^{2}} \quad ; \quad G_{E}^{n}=0
$$


for the charge form factors. The scale parameter in the distribution (3.2) is related to the proton charge radius $\left\langle r^{2}\right\rangle_{E}^{p}=12 a^{2}$. Similarly, for the magnetic form factors we obtain

$$
G_{M}^{p} / \mu_{p}=G_{M}^{n} / \mu_{n}=\frac{1}{\left(1+k^{2} a^{2}\right)^{2}},
$$

where the corresponding magnetic moments are

$$
\mu_{p}=\mu \quad ; \quad \mu_{n}=-\frac{2}{3} \mu
$$

respectively. Here we have assumed spin-flavor symmetry so that the mass and the $g$-factor of the up $(u)$ and down $(d)$ constituents are identical, $m_{u}=m_{d}=m_{q}$ and $g_{u}=g_{d}=g$. Accordingly $\mu_{u}=\mu_{d}=\mu$ and $\mu=e g / 2 m_{q}$ in Eq. (4.4). The corresponding harmonic oscillator elastic form factors are obtained by replacing the dipole function $\left(1+k^{2} a^{2}\right)^{-2}$ by the exponential function $e^{-k^{2} \beta^{2} / 6}$ with $\beta$ related to the harmonic oscillator size parameter. The form factors in Eqs. (4.2)-(4.3) (and their harmonic oscillator analogues) satisfy $G_{M}^{p}=\mu G_{E}^{p}$ and the relations $G_{E}^{n}=0$ and $G_{M}^{n} / G_{M}^{p}=-2 / 3$ for all values of the momentum transfer. These relations are due to spin-flavor symmetry, but are not obeyed by the experimental data. Within a truncated three-constituents configuration space, to have a nonvanishing neutron electric form factor, one must break $S U_{s f}(6)$ [1]. This breaking can be achieved in various ways, e.g. by including in the mass operator a hyperfine interaction [12], or by distorting the oblate-top geometry, allowing for a quark-diquark structure [13]. Within the model discussed here we study the breaking of the $S U_{s f}(6)$ symmetry by assuming a flavor-dependent distribution of the charge and the magnetization along the strings of Fig. 1,

$$
\begin{aligned}
& g_{u}(\beta)=\beta^{2} \mathrm{e}^{-\beta / a_{u}} / 2 a_{u}^{3}, \\
& g_{d}(\beta)=\beta^{2} \mathrm{e}^{-\beta / a_{d}} / 2 a_{d}^{3} .
\end{aligned}
$$

With this dependence and for small symmetry-breaking the electric nucleon form factors become

$$
\begin{aligned}
G_{E}^{p} & \approx \frac{1}{\left(1+k^{2} \bar{a}^{2}\right)^{2}}\left[1+\frac{5}{3} \frac{k^{2} \bar{a}^{2}}{\left(1+k^{2} \bar{a}^{2}\right)} \Delta\right], \\
G_{E}^{n} & \approx \frac{4}{3} \frac{k^{2} \bar{a}^{2}}{\left(1+k^{2} \bar{a}^{2}\right)^{3}} \Delta,
\end{aligned}
$$


to leading order in $\Delta$. Here $\Delta \equiv\left(a_{d}^{2}-a_{u}^{2}\right) / \bar{a}^{2}$ with $\bar{a}^{2}=\left(a_{d}^{2}+a_{u}^{2}\right) / 2$.

If the length of the string in Fig. 1 is slightly different for $u$ and $d$, so is their mass and thus in principle, their magnetic moment. Applying the same procedure to the magnetic form factors gives (to leading order in $\Delta$ )

$$
\begin{aligned}
G_{M}^{p} / \mu_{p} & \approx \frac{1}{\left(1+k^{2} \bar{a}^{2}\right)^{2}}\left\{1+\frac{k^{2} \bar{a}^{2}}{\left(1+k^{2} \bar{a}^{2}\right)}\left[\frac{7}{9}-\frac{16}{81}\left(\frac{\mu_{d}-\mu_{u}}{\mu_{p}}\right)\right] \Delta\right\} \\
G_{M}^{n} / \mu_{n} & \approx \frac{1}{\left(1+k^{2} \bar{a}^{2}\right)^{2}}\left\{1-\frac{k^{2} \bar{a}^{2}}{\left(1+k^{2} \bar{a}^{2}\right)}\left[\frac{1}{3}-\frac{8}{27}\left(\frac{\mu_{d}-\mu_{u}}{\mu_{n}}\right)\right] \Delta\right\} .
\end{aligned}
$$

Here $\mu_{u} e_{u}$ and $\mu_{d} e_{d}$ are the magnetic moments of the $u$ and $d$ constituents which determine the proton and neutron magnetic moments

$$
\mu_{p}=\left(4 \mu_{u} e_{u}-\mu_{d} e_{d}\right) / 3 \quad ; \quad \mu_{n}=\left(4 \mu_{d} e_{d}-\mu_{u} e_{u}\right) / 3
$$

In the calculations reported below we have used the following procedure to determine the parameters. In all cases we take $g_{u}=g_{d}=1$. We assume that the constituent masses $m_{u}$ and $m_{d}$ are determined from the measured magnetic moments. This fixes the scale magnetic moments $\mu_{u}$ and $\mu_{d}$. The scale parameters $a_{u}$ and $a_{d}$ in the distributions (4.5) are determined from a simultaneous fit to the proton and neutron charge radii, and to the proton and neutron electric and magnetic form factors. For the calculations in which the $S U_{s f}(6)$ symmetry is satisfied $\left(\mu_{u}=\mu_{d}=\mu\right.$ and $\left.a_{u}=a_{d}=a\right)$ this procedure yields $a=0.232 \mathrm{fm}$ and $\mu=\mu_{p}=2.793 \mu_{N}\left(=0.126 \mathrm{GeV}^{-1}\right)$, which corresponds to a constituent mass of $m_{u}=m_{d}=0.336 \mathrm{GeV}$. For the calculations in which the $S U_{s f}(6)$ symmetry is broken we find $a_{u}=0.230 \mathrm{fm}$ and $a_{d}=0.257 \mathrm{fm}$ (implying $\Delta=0.2$ in Eqs. (4.6)-(4.7) ), $\mu_{u}=2.777 \mu_{N}\left(=0.126 \mathrm{GeV}^{-1}\right), \mu_{d}=2.915 \mu_{N},\left(=0.133 \mathrm{GeV}^{-1}\right)$ corresponding to $m_{u}=0.338 \mathrm{GeV}$ and $m_{d}=0.322 \mathrm{GeV}$, respectively.

Fig. 4 shows the elastic electric form factors of the proton and the neutron divided by the dipole form, $F_{D}=1 /\left(1+Q^{2} / 0.71\right)^{2}$. The division by $F_{D}$ emphasizes the effect of the breaking of spin-flavor symmetry. Fig. 5 shows the results for the elastic magnetic form factors. We see that while the breaking of spin-flavor symmetry can account for the nonzero value of $G_{E}^{n}$ and gives a good description of the data, it worsens the fit to the proton electric and neutron magnetic form factors. This implies either that the simple mechanism 
for spin-flavor breaking of Eq. (4.5) does not produce the right phenomenology and other contributions, such as polarization of the neutron into $p+\pi^{-}$, play an important role in the neutron electric form factor [14]. (A coupling to the meson cloud through $\rho, \omega$ and $\phi$ mesons is indeed expected to contribute in this range of $Q^{2}$ [15].) This conclusion (i.e. worsening the proton form factors) applies also to the other mechanisms of spin-flavor symmetry breaking mentioned above, such as that induced by a hyperfine interaction 12] which gives $a_{u}<a_{d}$ ('moves the up quark to the center and the down quark to the periphery'). This pattern is a consequence of the fact that within the framework of constituent models $G_{E}^{p}, G_{E}^{n}, G_{M}^{p}$ and $G_{M}^{n}$ are intertwined as is clearly evident from the expressions in Eqs. (4.6)-(4.7).

\subsection{Helicity Amplitudes}

Other (observable) quantities of interest are the helicity amplitudes in photo- and electroproduction. The transverse helicity amplitudes between the initial (ground) state of the nucleon and the final (excited) state of a baryon resonance are expressed as [5]

$$
A_{\nu}^{N}=6 \sqrt{\frac{\pi}{k_{0}}}[k\langle L, 0 ; S, \nu \mid J, \nu\rangle \mathcal{B}-\langle L, 1 ; S, \nu-1 \mid J, \nu\rangle \mathcal{A}],
$$

where $\nu=1 / 2,3 / 2$ indicates the helicity. The orbit- and spin-flip amplitudes $(\mathcal{A}$ and $\mathcal{B}$, respectively) are given by

$$
\begin{aligned}
& \mathcal{B}=\int \mathrm{d} \beta g(\beta)\left\langle\Psi_{f} ; M_{J}=\nu\left|\mu_{3} e_{3} s_{3,+} \hat{U}\right| \Psi_{i} ; M_{J}^{\prime}=\nu-1\right\rangle, \\
& \mathcal{A}=\int \mathrm{d} \beta g(\beta)\left\langle\Psi_{f} ; M_{J}=\nu\left|\mu_{3} e_{3} \hat{T}_{+} / g_{3}\right| \Psi_{i} ; M_{J}^{\prime}=\nu-1\right\rangle .
\end{aligned}
$$

These observables correspond to an absorption process $\left(\gamma+B^{\prime} \rightarrow B\right)$ of a right-handed photon with four-momentum $\left(k_{0}, \vec{k}=k \hat{z}\right)$. In Eq. (4.10) $\left|\Psi_{i}\right\rangle$ denotes the (space-spinflavor) wave function of the initial nucleon $\left(B^{\prime}\right)$ with ${ }^{2} 8_{1 / 2}^{N}\left[56,0^{+}\right]_{(0,0) ; 0}$ and $N=p, n$, and, similarly, $\left|\Psi_{f}\right\rangle$ that of the final baryon resonance $(B)$. In general, the $\mathcal{B}$ and $\mathcal{A}$ amplitudes of Eq. (4.10) are proportional to the collective form factors $\mathcal{F}$ and $\mathcal{G}_{+}$of Eq. (3.3), respectively. The breaking of spin-flavor symmetry has also influence on the helicity amplitudes of Eq. (4.9). They would now be given in terms of flavor-dependent collective 
form factors $\mathcal{F}_{u}(k), \mathcal{G}_{u,+}(k)$ and $\mathcal{F}_{d}(k), \mathcal{G}_{d,+}(k)$, which depend on the size parameters, $a_{u}$ and $a_{d}$, respectively.

When comparing with the experimental data one must still choose a reference frame which determines the relation between the three-momentum $k^{2}$ and the four-momentum $Q^{2}=k^{2}-k_{0}^{2}$. It is convenient to choose the equal momentum or Breit frame where

$$
k^{2}=Q^{2}+\frac{\left(W^{2}-M^{2}\right)^{2}}{2\left(M^{2}+W^{2}\right)+Q^{2}} .
$$

Here $M$ is the nucleon mass, $W$ is the mass of the resonance, and $-Q^{2}$ can be interpreted as the mass squared of the virtual photon (for elastic scattering we have $k^{2}=Q^{2}$ ). In assessing the quality of the fits it should be noted that there are no adjustable parameters involved in the calculation of helicity amplitudes. All the parameters appearing in Eq. (4.10) and in the distributions (4.5) are those extracted previously from the elastic form factors.

Representative results for transverse helicity amplitudes $A_{1 / 2}$ and $A_{3 / 2}$ are shown in Fig. 6 for the nucleon resonances $N(1520) D_{13}$ and $N(1680) F_{15}$. From this figure it is seen that the effect of spin-flavor breaking is rather small. Only in those cases in which the amplitude with $S U_{s f}(6)$ symmetry is zero, the effect is of some relevance. Such is the case with proton helicity amplitudes for the ${ }^{4} 8_{J}\left[70, L^{P}\right]$ multiplet (e.g. the $L^{P}=1^{-}$ resonances $N(1675) D_{15}$ and $\left.N(1700) D_{13}\right)$ and with neutron helicity-3/2 amplitudes for the ${ }^{2} 8_{J}\left[56, L^{P}\right]$ multiplet (e.g. the $L^{P}=2^{+}$resonance $N(1680) F_{15}$ ). The conclusion that one can draw from this analysis is that, for all purposes, with the exception of the electric form factor of the neutron, the breaking of spin-flavor symmetry according to the mechanism of Eq. (4.5) is of little importance.

In a string-like model of hadrons one expects on the basis of QCD [16 that strings will elongate (hadrons swell) as their energy increases. This effect can be easily included in the present analysis by making the scale parameters of the strings energy dependent. We use here the simple ansatz

$$
a=a_{0}\left(1+\xi \frac{W-M}{M}\right)
$$


where $M$ is the nucleon mass and $W$ the resonance mass. This ansatz introduces a new parameter $(\xi)$, the stretchability of the string.

Figs. 7 shows the effect of stretching on the helicity amplitudes for $N(1520) D_{13}$ and $N(1680) F_{15}$. It is seen that the effect of stretching, especially if one takes the value $\xi \approx 1$ suggested by the arguments of [16] and the Regge behavior of nucleon resonances (see e.g. Fig. 5 of [5]), is rather large. In particular, the data for $N(1680) F_{15}$ and $N(1520) D_{13}$ show a clear indication that the form factors are dropping faster than expected on the basis of the dipole form. (Of course for the elastic form actors there is no stretching.) We suggest that future data at CEBAF and MAMI be used to analyze the effects of stretching on the helicity amplitudes.

\section{Strong Couplings}

We consider in this Section strong decays of baryons of the form $B \rightarrow B^{\prime}+M$ [8]. The process involves an emission (by one of the constituents in $B$ ) of an elementary pseudoscalar meson meson $(M=\pi$ or $\eta)$ with energy $k_{0}=E_{M}=E_{B}-E_{B^{\prime}}$ and momentum $\vec{k}=\vec{P}_{M}=\vec{P}-\vec{P}^{\prime}=k \hat{z}$. Here $\vec{P}=P_{z} \hat{z}$ and $\vec{P}^{\prime}\left(=P_{z}^{\prime} \hat{z}\right)$ are the momenta of the initial $(B)$ and final baryon $\left(B^{\prime}\right)$. The calculations are performed in the rest frame of $B\left(P_{z}=0\right)$.

In the collective model the strong couplings (similar to the electromagnetic couplings) are obtained by folding the matrix elements of the transition operator inducing the decay with the distribution function $g(\beta)$ of Eq. (3.2). These collective matrix elements can be expressed in terms of helicity amplitudes. For decays in which the initial baryon has

angular momentum $\vec{J}=\vec{L}+\vec{S}$ and in which the final baryon is either the nucleon or the delta with wave functions (2.2), the (strong) helicity amplitudes are

$$
\begin{aligned}
A_{\nu}(k)= & \frac{1}{(2 \pi)^{3 / 2}\left(2 k_{0}\right)^{1 / 2}}\left[\langle L, 0, S, \nu \mid J, \nu\rangle \zeta_{0} Z_{0}(k)+\frac{1}{2}\langle L, 1, S, \nu-1 \mid J, \nu\rangle \zeta_{+} Z_{-}(k)\right. \\
& \left.+\frac{1}{2}\langle L,-1, S, \nu+1 \mid J, \nu\rangle \zeta_{-} Z_{+}(k)\right] .
\end{aligned}
$$

The coefficients $\zeta_{m}$ are spin-flavor matrix elements [8] and $Z_{m}(k)(m=0, \pm)$ are the radial 
matrix elements

$$
\begin{aligned}
Z_{0}(k) & =6\left[g k-\frac{1}{6} h k\right] \mathcal{F}(k)^{*}-6 h \mathcal{G}_{z}(k)^{*} \\
Z_{ \pm}(k) & =-6 h \mathcal{G}_{\mp}(k)^{*}
\end{aligned}
$$

The coefficients $g$ and $h$ denote the strength of two terms in the transition operator. The radial matrix elements $Z_{m}(k)$ involve the same collective form factors $\mathcal{F}(k), \mathcal{G}_{\mp}(k)$ discussed in Section (3). The reason for the complex conjugation in Eq. (5.2) is that here we consider an emission process, whereas Table 1 shows the form factors for an absorption process.

The decay widths for a specific channel are given by [17]

$$
\Gamma\left(B \rightarrow B^{\prime}+M\right)=2 \pi \rho_{f} \frac{2}{2 J+1} \sum_{\nu>0}\left|A_{\nu}(k)\right|^{2}
$$

where $\rho_{f}$ is a phase space factor. For all resonances with the same value of $\left(v_{1}, v_{2}\right), L^{P}$ this expression can be rewritten in a more transparent form in terms of only two elementary partial wave amplitudes $W_{l}(k)$,

$$
\Gamma\left(B \rightarrow B^{\prime}+M\right)=2 \pi \rho_{f} \frac{1}{(2 \pi)^{3} 2 k_{0}} \sum_{l=L \pm 1} c_{l}\left|W_{l}(k)\right|^{2}
$$

Here $l$ is the relative orbital angular momentum between the final baryon and the emitted meson. It takes the values $l=L \pm 1$ (the value $l=L$ is not allowed because of parity conservation). For this set of resonances, the $k$ dependence is contained in the partial wave amplitudes $W_{l}(k)$, while the dependence on the individual baryon resonance is contained in the coefficients $c_{l}$. In the algebraic method, the $W_{l}(k)$ can be obtained in closed form. For example, the corresponding $S$ and $D$ elementary partial wave amplitudes are

$$
\begin{aligned}
& W_{0}(k)=i\left\{\left[g k-\frac{1}{6} h k\right] \frac{k a}{\left(1+k^{2} a^{2}\right)^{2}}+h m_{3} k_{0} a \frac{3-k^{2} a^{2}}{\left(1+k^{2} a^{2}\right)^{3}}\right\}, \\
& W_{2}(k)=i\left\{\left[g k-\frac{1}{6} h k\right] \frac{k a}{\left(1+k^{2} a^{2}\right)^{2}}-h m_{3} k_{0} a \frac{4 k^{2} a^{2}}{\left(1+k^{2} a^{2}\right)^{3}}\right\} .
\end{aligned}
$$

Partial widths for other models of the nucleon and its resonances can be obtained by introducing the corresponding expressions for the elementary amplitudes $W_{l}(k)$. For 
example, the relevant expressions in the harmonic oscillator quark model are

$$
\begin{aligned}
& W_{0}(k)=\frac{i}{3}\left\{\left[g k-\frac{1}{6} h k\right] k \beta+h m_{3} k_{0} \beta\left(3-\frac{k^{2} \beta^{2}}{3}\right)\right\} \mathrm{e}^{-k^{2} \beta^{2} / 6}, \\
& W_{2}(k)=\frac{i}{3}\left\{\left[g k-\frac{1}{6} h k\right] k \beta-\frac{1}{3} h m_{3} k_{0} \beta k^{2} \beta^{2}\right\} \mathrm{e}^{-k^{2} \beta^{2} / 6},
\end{aligned}
$$

Use of Eqs. (5.4)-(5.5) allows us to do a straightforward and systematic analysis of the experimental data. The calculations are performed in the rest frame of the decaying resonance, in which the relativistic expression for the phase space factor $\rho_{f}$ as well as for the momentum $k$ of the emitted meson are retained. The expressions for $k$ and $\rho_{f}$ are

$$
\begin{aligned}
k^{2} & =-m_{M}^{2}+\frac{\left(m_{B}^{2}-m_{B^{\prime}}^{2}+m_{M}^{2}\right)^{2}}{4 m_{B}^{2}}, \\
\rho_{f} & =4 \pi \frac{E_{B^{\prime}}(k) E_{M}(k) k}{m_{B}}
\end{aligned}
$$

with $E_{B^{\prime}}(k)=\sqrt{m_{B^{\prime}}^{2}+k^{2}}$ and $E_{M}(k)=\sqrt{m_{M}^{2}+k^{2}}$.

We consider here decays with emission of $\pi$ and $\eta$. The calculated values depend on the two parameters $g$ and $h$ in Eq. (5.2) and on the scale parameter $a$ of Eq. (3.2). In the present analysis we determine these parameters from a least square fit to the $N \pi$ partial widths (which are relatively well known) with the exclusion of the $S_{11}$ resonances. For the latter the situation is not clear due to possible mixing of $N(1535) S_{11}$ and $N(1650) S_{11}$ and the possible existence of a third $S_{11}$ resonance [18]. As a result we find $g=1.164$ $\mathrm{GeV}^{-1}$ and $h=-0.094 \mathrm{GeV}^{-1}$. The relative sign is consistent with a previous analysis of the strong decay of mesons [19] and with a derivation from the axial-vector coupling (see e.g. [17]). The scale parameter, $a=0.232 \mathrm{fm}$, extracted in the present fit is found to be equal to the value extracted in the calculation of electromagnetic couplings [7]. We keep $g, h$ and $a$ equal for all resonances and all decay channels $(N \pi, N \eta, \Delta \pi, \Delta \eta)$. In comparing with previous calculations, it should be noted that in the calculation in the nonrelativistic quark model of [20] the decay widths are parametrized by four reduced partial wave amplitudes instead of the two elementary amplitudes $g$ and $h$. Furthermore, the momentum dependence of these reduced amplitudes are represented by constants. The calculation in the relativized quark model of [21] was done using a pair-creation 
model for the decay and involved a different assumption on the phase space factor. Both the nonrelativistic and relativized quark model calculations include the effects of mixing induced by the hyperfine interaction, which in the present calculation are not taken into account.

The calculations of decay widths of $3^{*}$ and $4^{*}$ resonances into the $N \pi$ and $\Delta \pi$ channels are in fair agreement with experiment. Representative results are shown in Fig. 8. The results are to a large extent a consequence of spin-flavor symmetry. The use of 'collective' form factors improves somewhat the results when compared with older (harmonic oscillator) calculations. This is shown in Table $\mathbb{1}$ where the decay of a $\Delta$ Regge trajectory into $N \pi$ is analyzed and compared with the calculations of [17], which are based on the harmonic oscillator model discussed in [22]. We also include the results of more recent calculations in the nonrelativistic quark model [20] and in the relativized quark model [21. There does not seem to be anything unusual in the decays into $\pi$ and our analysis confirms the results of previous analyses.

Contrary to the decays into $\pi$, the decay widths into $\eta$ have some unusual properties. The calculation gives systematically small values for these widths. This is due to a combination of phase space factors and the structure of the transition operator. Both depend on the momentum transfer $k$, however, due to the difference between the $\pi$ and $\eta$ mass, the momentum carried by the $\eta$ is smaller than that carried by the $\pi$. Therefore, the $\eta$ decay widths are suppressed relative to the $\pi$ decays. The spin-flavor part is approximately the same for $N \pi$ and $N \eta$, since $\pi$ and $\eta$ are in the same $S U_{f}(3)$ multiplet. We emphasize here, that the transition operator was determined by fitting the coefficients $g$ and $h$ to the $N \pi$ decays of the $3^{*}$ and $4^{*}$ resonances. Hence the $\eta$ decays are calculated without introducing any further parameters.

The experimental situation is unclear. The 1992 Particle Data Group (PDG) compilation gave systematically small widths $(\sim 1 \mathrm{MeV})$ for all resonances except $N(1535) S_{11}$. The 1994 PDG compilation deleted all $\eta$ widths with the exception of $N(1535) S_{11}$. This situation persists in the latest PDG compilation [23], where $N(1650) S_{11}$ is now assigned a small but non-zero $\eta$ width. The results of our analysis suggest that the large $\eta$ width 
for the $N(1535) S_{11}$ is not due to a conventional $q^{3}$ state. One possible explanation is the presence of another state in the same mass region, e.g. a quasi-bound meson-baryon $S$ wave resonance just below or above threshold, for example $N \eta, K \Sigma$ or $K \Lambda$ [24]. Another possibility is an exotic configuration of four quarks and one antiquark $\left(q^{4} \bar{q}\right)$.

\section{Summary and conclusions}

In this article, we have exploited the algebraic approach to baryon structure to analyze simultaneously elastic form factors and helicity amplitudes in photo- and electroproduction and strong decay widths. The use of algebraic methods allows us to study different situations, such as the harmonic oscillator quark model and the collective model, within the same framework. The logic of the method is that, by starting from the charge and magnetization distribution of the ground state (assuming a dipole form to the elastic form factor of the nucleon), one can obtain the transition form factors to the excited states. In the 'collective' model, this procedure yields a power dependence of all form factors (elastic and inelastic) on $Q^{2}$. For electromagnetic couplings we have analyzed two aspects of hadronic structure: (i) the breaking of $S U_{s f}(6)$ symmetry, and (ii) the stretching of hadrons with increasing excitation energy. We find that, whereas the breaking of the spin-flavor symmetry hardly effects the helicity amplitudes, the stretching of hadrons does have a noticeable influence.

The disagreement between experimental and theoretical elastic form factors and he-

licity amplitudes in the low- $Q^{2}$ region $0 \leq Q^{2} \leq 1(\mathrm{GeV} / \mathrm{c})^{2}$ may be due to coupling of the photon to the meson cloud, (i.e. configurations of the type $q^{3}-q \bar{q}$ ). Since such configurations have much larger spatial extent than $q^{3}$, their effects are expected to drop faster with momentum transfer $Q^{2}$ than the constituent form factors. Also, since meson exchange corrections contribute differently to different channels, this effect will be state dependent.

We have performed a calculation of the strong decay widths $N^{*} \rightarrow N+\pi, N^{*} \rightarrow \Delta+\pi$, $N^{*} \rightarrow N+\eta, \Delta^{*} \rightarrow N+\pi, \Delta^{*} \rightarrow \Delta+\pi$ and $\Delta^{*} \rightarrow \Delta+\eta$ in a collective model of baryons. 
The analysis of experimental data shows that, while the decays into $\pi$ follow the expected pattern, the decays into $\eta$ have some unusual features. Our calculations do not show any indication for a large $\eta$ width, as is observed for the $N(1535) S_{11}$ resonance. The observed large $\eta$ width indicates the presence of another configuration, which is outside the present model space. This suggests, that in order to elucidate this point, particular attention be paid at CEBAF to the $N \eta$ channel.

In the present contribution we have focused the discussion to the nonstrange sector (nucleon and delta). It will be of interest to extend the formalism to include strange baryons as well.

\section{Acknowledgements}

The results reported in these proceedings are based on work done in collaboration with F. Iachello (Yale). The work is supported in part by grant No. 94-00059 from the United States-Israel Binational Science Foundation (BSF), Jerusalem, Israel (A.L.) and by CONACyT, México under project 400340-5-3401E and DGAPA-UNAM under project IN105194 (R.B.). 


\section{References}

[1] M. Gell-Mann, Phys. Rev. 125, 1067 (1962); Y. Ne'eman, Nucl. Phys. 26, 222 (1961).

[2] F. Gürsey and L.A. Radicati, Phys. Rev. Lett. 13, 173 (1964).

[3] N. Isgur and G. Karl, Phys. Rev. D18, 4187 (1978); D19, 2653 (1979); D20, 1191 (1979).

[4] S. Capstick and N. Isgur, Phys. Rev. D34, 2809 (1986).

[5] R. Bijker, F. Iachello and A. Leviatan, Ann. Phys. (N.Y.) 236, 69 (1994).

[6] R. Bijker and A. Leviatan, these proceedings.

[7] R. Bijker, F. Iachello and A. Leviatan, Phys. Rev. C54, 1935 (1996).

[8] R. Bijker, F. Iachello and A. Leviatan, preprint nucl-th/9608057, Phys. Rev. D, in press.

[9] R. Bijker and A. Leviatan, in "Symmetries in Science VII: Spectrum Generating Algebras and Dynamic Symmetries in Physics", eds. B. Gruber and T. Otsuka, Plenum Press, New York, 87 (1993); R. Bijker and A. Leviatan, Rev. Mex. Fís. 39, Suplemento 2, 7 (1993); ibid. 42 Suplemento 2, 62 (1995); A. Leviatan and R. Bijker, $\pi N$ Newsletter 11, 142 (1995).

[10] N. Isgur, G. Karl and R. Koniuk, Phys. Rev. Lett. 41, 1269 (1978).

[11] J.L. Friar, Part. and Nucl. 4, 153 (1972);

R.D. Carlitz, S.D. Ellis and R. Savit, Phys. Lett. B68, 443 (1977).

[12] N. Isgur, G. Karl and D.W.L. Sprung, Phys. Rev. D23, 163 (1981).

[13] Y. Tzeng and S.S. Hsiao, Il Nuovo Cimento 106, 573 (1993). 
[14] F.E. Close and R.R. Hogan, Nucl. Phys. B185, 333 (1981);

O.V. Mawell and V. Vento. Nucl. Phys. A407, 366 (1983);

S. Theberge, A.W. Thomas and G.A. Miller, Phys. Rev. D22, 2838 (1980).

[15] F. Iachello, A.D. Jackson and A. Lande, Phys. Lett. B43, 191 (1973).

G. Höhler, E. Pietarinen, I. Sabba-Stefanescu, F. Borkowski, G.G. Simon, V.H. Walther and R.D. Wendling, Nucl. Phys. B114, 505 (1976).

[16] K. Johnson and C.B. Thorn, Phys. Rev. D13, 1934 (1974); I. Bars and H.J. Hanson, Phys. Rev. D13, 1744 (1974).

[17] A. Le Yaouanc, L. Oliver, O. Pène and J.-C. Raynal, 'Hadron transitions in the quark model', Gordon and Breach (1988).

[18] Z. Li and R. Workman, Phys. Rev. C53, R549 (1996).

[19] C. Gobbi, F. Iachello and D. Kusnezov, Phys. Rev. D50, 2048 (1994).

[20] R. Koniuk and N. Isgur, Phys. Rev. Lett. 44, 845 (1980); Phys. Rev. D21, 1868 (1980).

[21] S. Capstick and W. Roberts, Phys. Rev. D47, 1994 (1993); ibid. D49, 4570 (1994).

[22] R.H. Dalitz, in 'Quarks and Hadronic Structure', Ed. M. Morpurgo, Plenum (1977).

[23] Particle Data Group, Phys. Rev. D54, 1 (1996).

[24] N. Kaiser, P.B. Siegel and W. Weise, Phys. Lett. B362, 23 (1995). 


\section{Figure Captions}

Fig. 1 Collective model of baryons (the charge distribution of the proton is shown as an example.

Fig. 2 Nucleon and delta mass spectrum $\left(M\right.$ vs. $\left.J^{P}\right)$. Collective model [5] $(+)$, nonrelativistic quark model [3] $(\times)$, relativized quark model [4] $(\diamond)$.

Fig. 3 Probability distribution of a typical ground state collective oblate-top ground state wave function in an harmonic oscillator basis.

Fig. 4 Comparison between the experimental proton $\left(G_{E}^{p}\right)$ and neutron $\left(G_{E}^{n}\right)$ electric form factors with the corresponding collective form factors. Dashed (solid) lines correspond to a calculation with (without) flavor breaking. The form factors are divided by the dipole form factor, $F_{D}=1 /\left(1+Q^{2} / 0.71\right)^{2}$.

Fig. 5 Comparison between the experimental proton $\left(G_{M}^{p} / \mu_{p}\right)$ and neutron $\left(G_{M}^{n} / \mu_{n}\right)$ magnetic form factors with the corresponding collective form factors. Dashed (solid) lines correspond to a calculation with (without) flavor breaking. The form factors are divided by the dipole form factor, $F_{D}=1 /\left(1+Q^{2} / 0.71\right)^{2}$.

Fig. 6 Proton helicity amplitudes for excitation of $N(1520) D_{13}$ (a factor of $+i$ is suppressed) and $N(1680) F_{15}$. The calculation with and without flavor breaking are shown by dashed and solid lines, respectively.

Fig. 7 Effect of hadron swelling for excitation of $N(1520) D_{13}$ (a factor of $+i$ is suppressed) and $N(1680) F_{15}$. The curves are labelled by the value of the stretching parameter $\xi$ of Eq. (4.12).

Fig. 8 Strong decay widths for $\Delta^{*} \rightarrow N+\pi$ and $\Delta^{*} \rightarrow \Delta+\pi$ decays of positive parity resonances with $L^{P}=2^{+}$and negative parity resonances with $L^{P}=1^{-}$. The theoretical values are in parenthesis. All values in $\mathrm{MeV}$. 

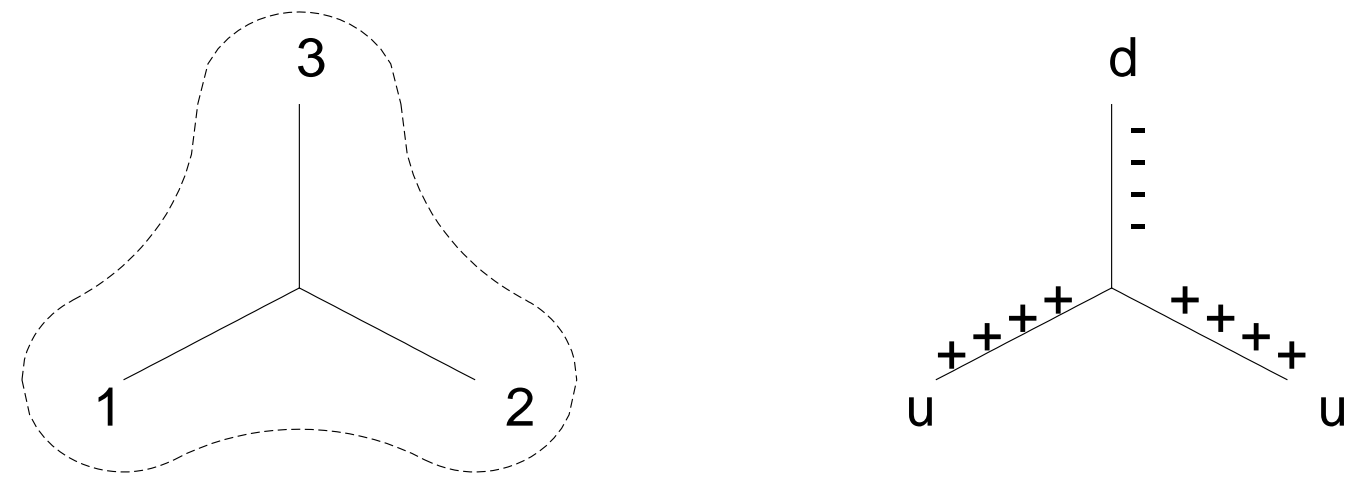

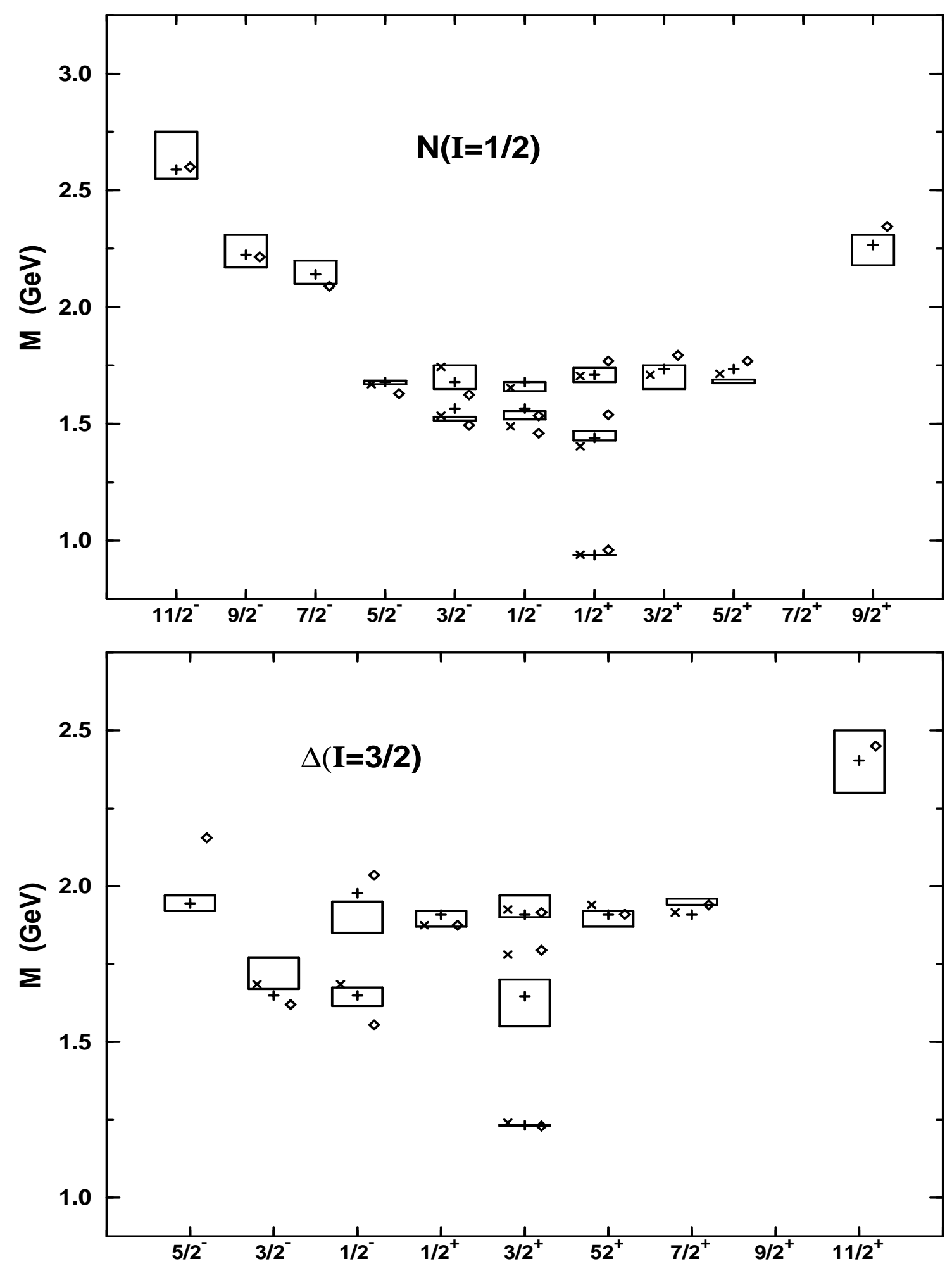


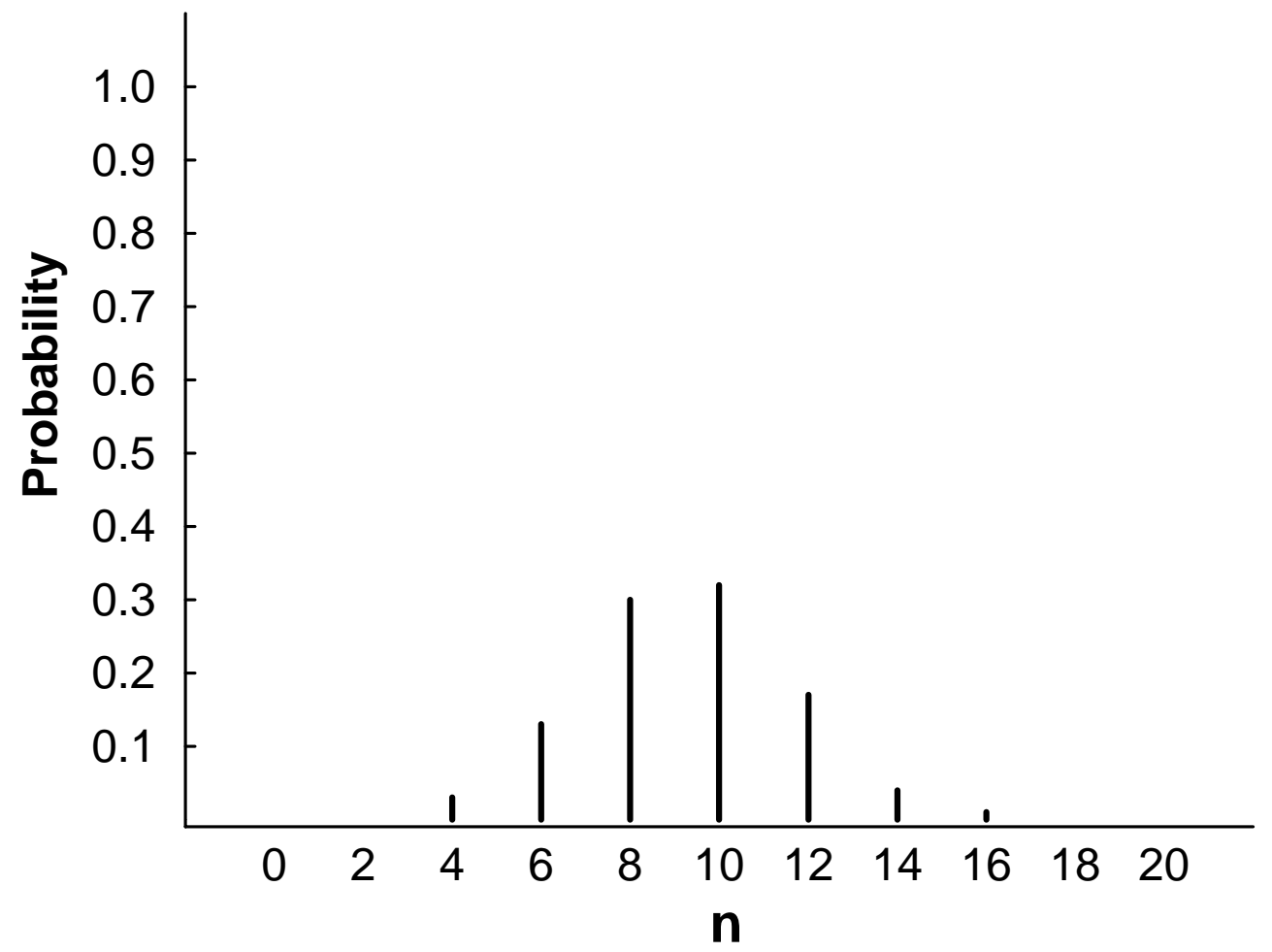



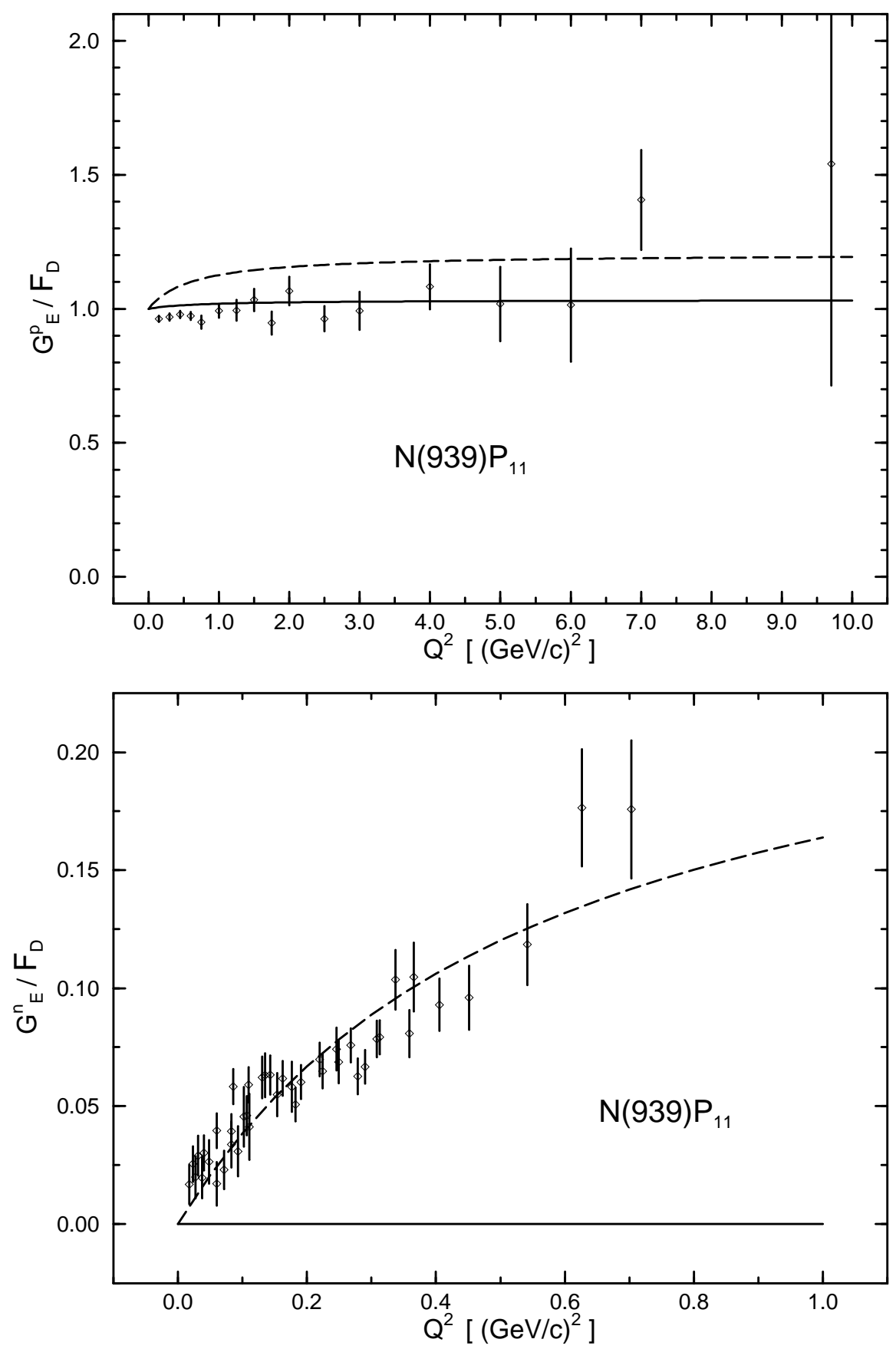

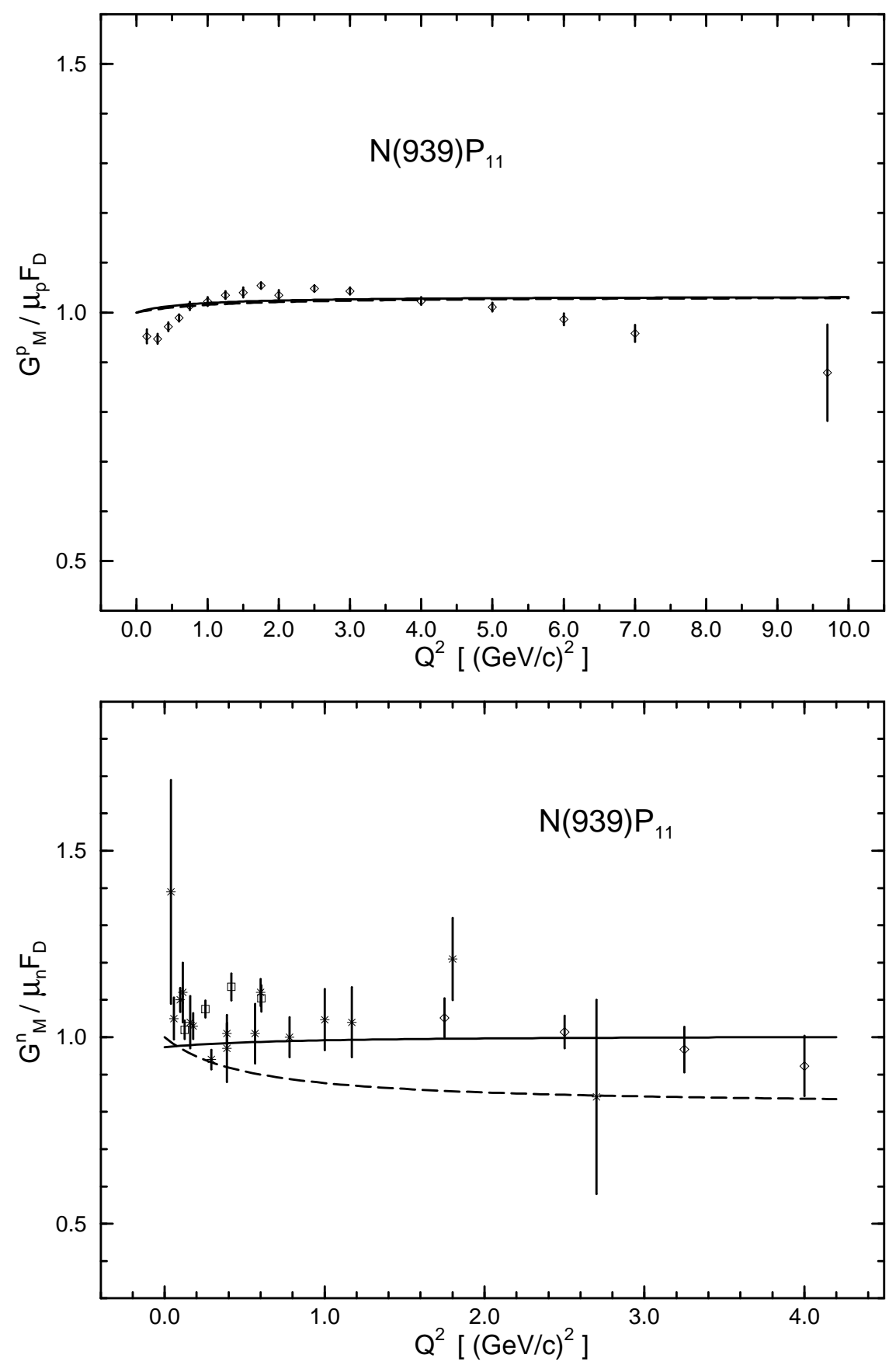

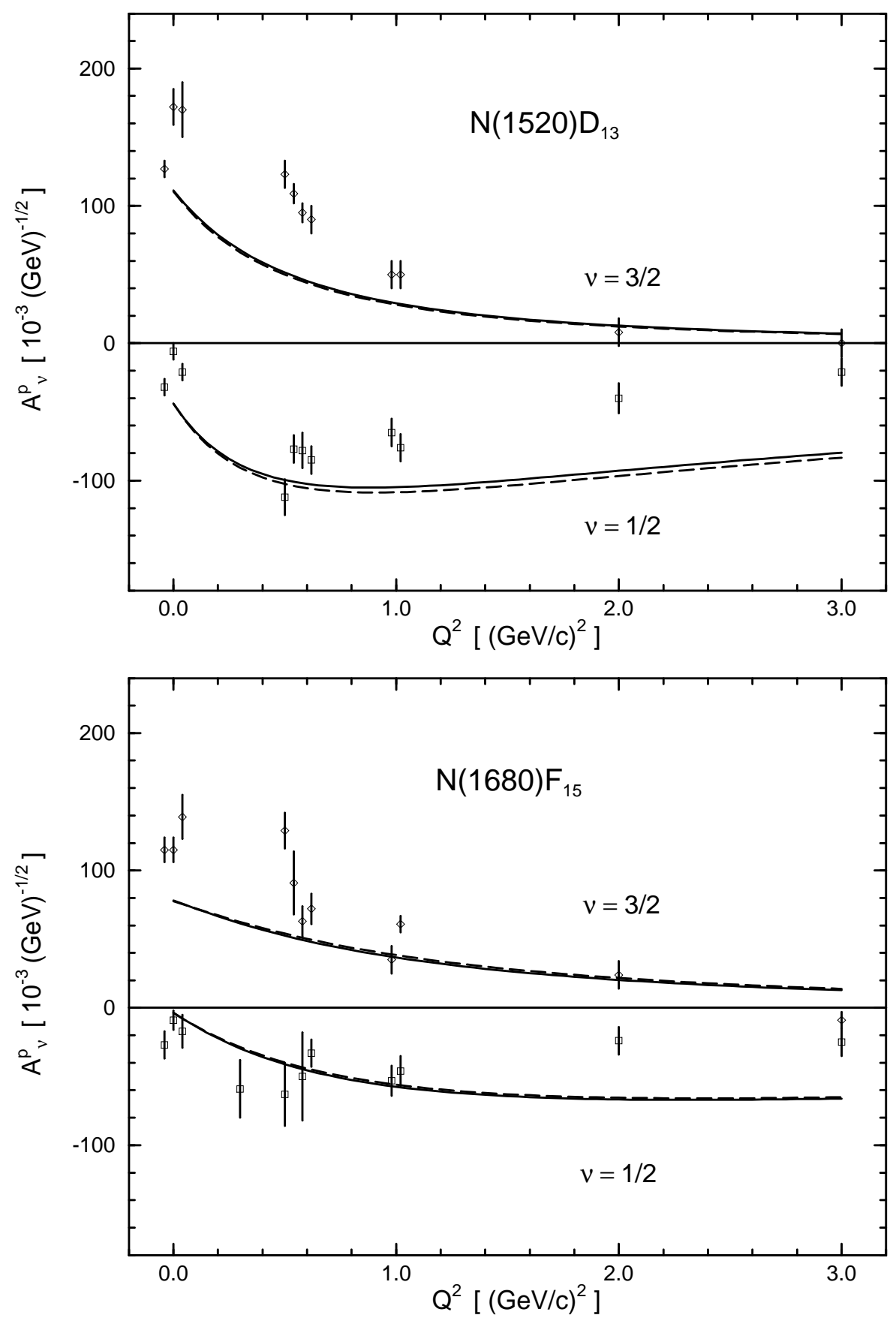

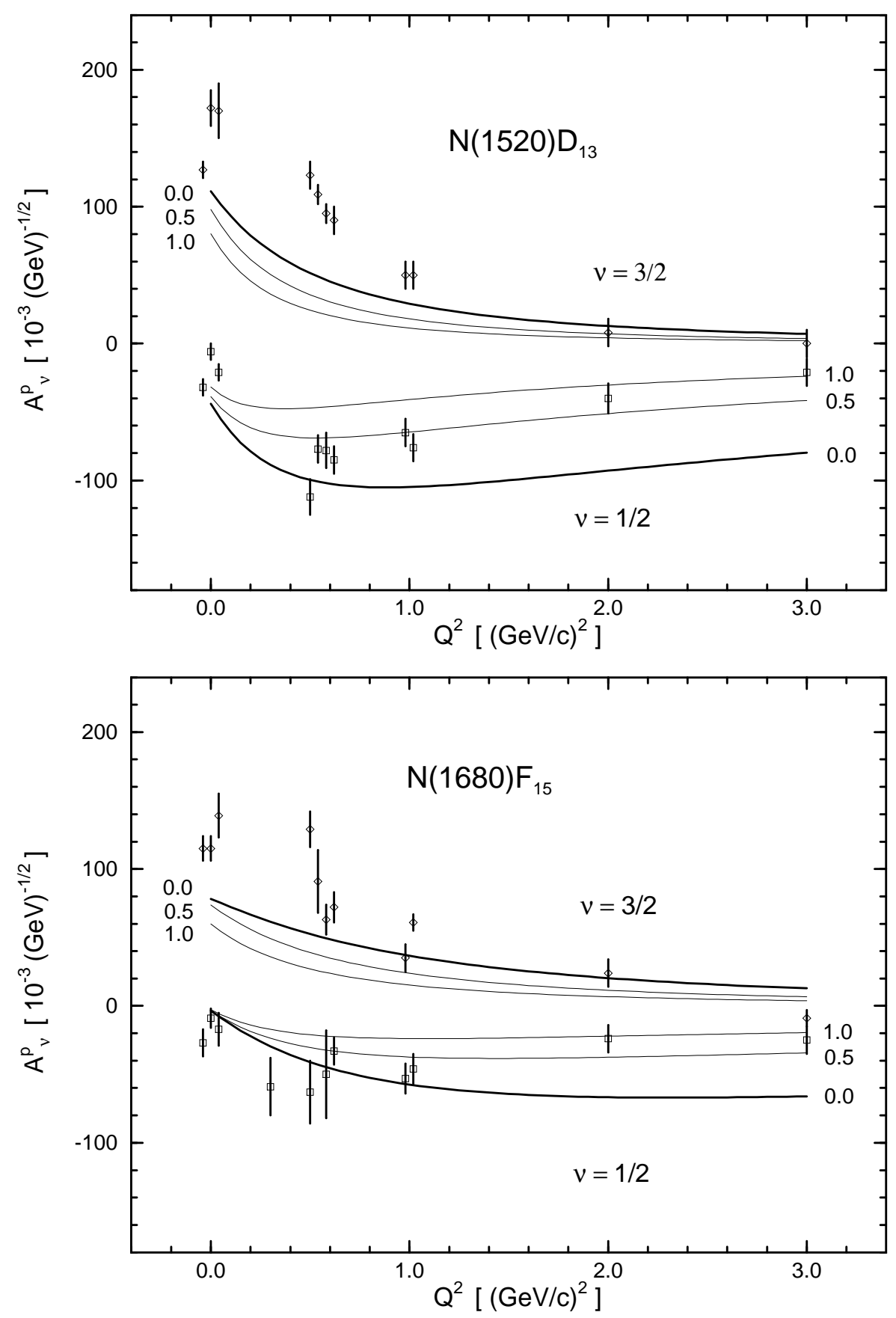


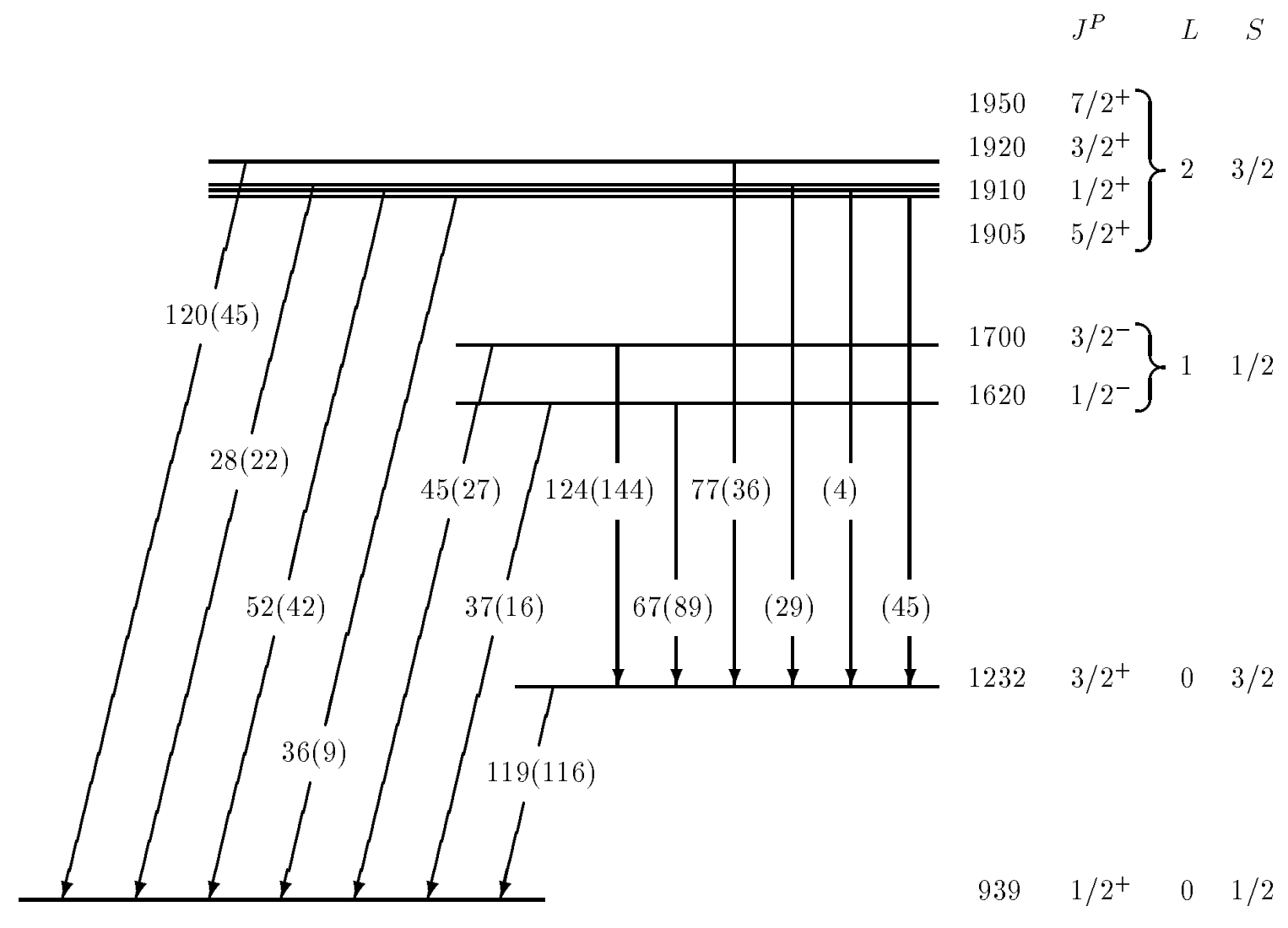


Table I: a) Collective form factors (for large model space). The final states are labeled by $\left[\operatorname{dim}\left\{S U_{s f}(6)\right\}, L^{P}\right]_{\left(v_{1}, v_{2}\right) ; K}$. The initial state is $\left[56,0^{+}\right]_{(0,0) ; 0} . H(x)=\arctan x-x /\left(1+x^{2}\right)$. b) Harmonic oscillator form factors. The final states are labeled by $\left[\operatorname{dim}\left\{S U_{s f}(6)\right\}, L^{P}\right]_{n}$. The initial state is $\left[56,0^{+}\right]_{0}$.
Final state ${ }^{a)}$
$\mathcal{F}(k)$
$\mathcal{G}_{z}(k) / m_{3} k_{0} a$
$\mathcal{G}_{ \pm}(k) / m_{3} k_{0} a$

$\begin{array}{rccc}{\left[56,0^{+}\right]_{(0,0) ; 0}} & \frac{1}{\left(1+k^{2} a^{2}\right)^{2}} & \frac{4 k a}{\left(1+k^{2} a^{2}\right)^{3}} & 0 \\ {\left[70,1^{-}\right]_{(0,0) ; 1}} & -i \sqrt{3} \frac{k a}{\left(1+k^{2} a^{2}\right)^{2}} & i \sqrt{3} \frac{1-3 k^{2} a^{2}}{\left(1+k^{2} a^{2}\right)^{3}} & \mp i \sqrt{6} \frac{1}{\left(1+k^{2} a^{2}\right)^{2}} \\ {\left[56,2^{+}\right]_{(0,0) ; 0}} & \frac{1}{2} \sqrt{5}\left[\frac{-1}{\left(1+k^{2} a^{2}\right)^{2}}\right. & -\frac{1}{2} \sqrt{5}\left[\frac{3+7 k^{2} a^{2}}{k a\left(1+k^{2} a^{2}\right)^{3}}\right. & \pm \sqrt{\frac{15}{2}}\left[\frac{-1}{k a\left(1+k^{2} a^{2}\right)^{2}}\right. \\ & \left.+\frac{3}{2 k^{3} a^{3}} H(k a)\right] & \left.-\frac{9}{2 k^{4} a^{4}} H(k a)\right] & \left.+\frac{3}{2 k^{4} a^{4}} H(k a)\right]\end{array}$

Final state ${ }^{b)}$

$\langle f|\hat{U}| i\rangle$

$\left\langle f\left|\hat{T}_{z}\right| i\right\rangle / m_{3} k_{0} \beta$

$\left\langle f\left|\hat{T}_{ \pm}\right| i\right\rangle / m_{3} k_{0} \beta$
$\left[56,0^{+}\right]_{0}$
$\mathrm{e}^{-k^{2} \beta^{2} / 6}$
$\frac{1}{3} k \beta \mathrm{e}^{-k^{2} \beta^{2} / 6}$
0
$\left[70,1^{-}\right]_{1}$
$-i \frac{1}{\sqrt{3}} k \beta \mathrm{e}^{-k^{2} \beta^{2} / 6}$
$i \frac{1}{\sqrt{3}}\left(1-\frac{k^{2} \beta^{2}}{3}\right) \mathrm{e}^{-k^{2} \beta^{2} / 6}$
$\mp i \sqrt{\frac{2}{3}} \mathrm{e}^{-k^{2} \beta^{2} / 6}$
$\left[56,2^{+}\right]_{2}$
$-\frac{1}{3 \sqrt{6}} k^{2} \beta^{2} \mathrm{e}^{-k^{2} \beta^{2} / 6}$
$\frac{2}{3 \sqrt{6}} k \beta\left(1-\frac{k^{2} \beta^{2}}{6}\right) \mathrm{e}^{-k^{2} \beta^{2} / 6}$
$\mp \frac{1}{3} k \beta \mathrm{e}^{-k^{2} \beta^{2} / 6}$ 
Table II: Strong decay widths for $\Delta^{*} \rightarrow N+\pi$ and $N^{*} \rightarrow N+\pi$ in $\mathrm{MeV}$. Experimental values are from [23].

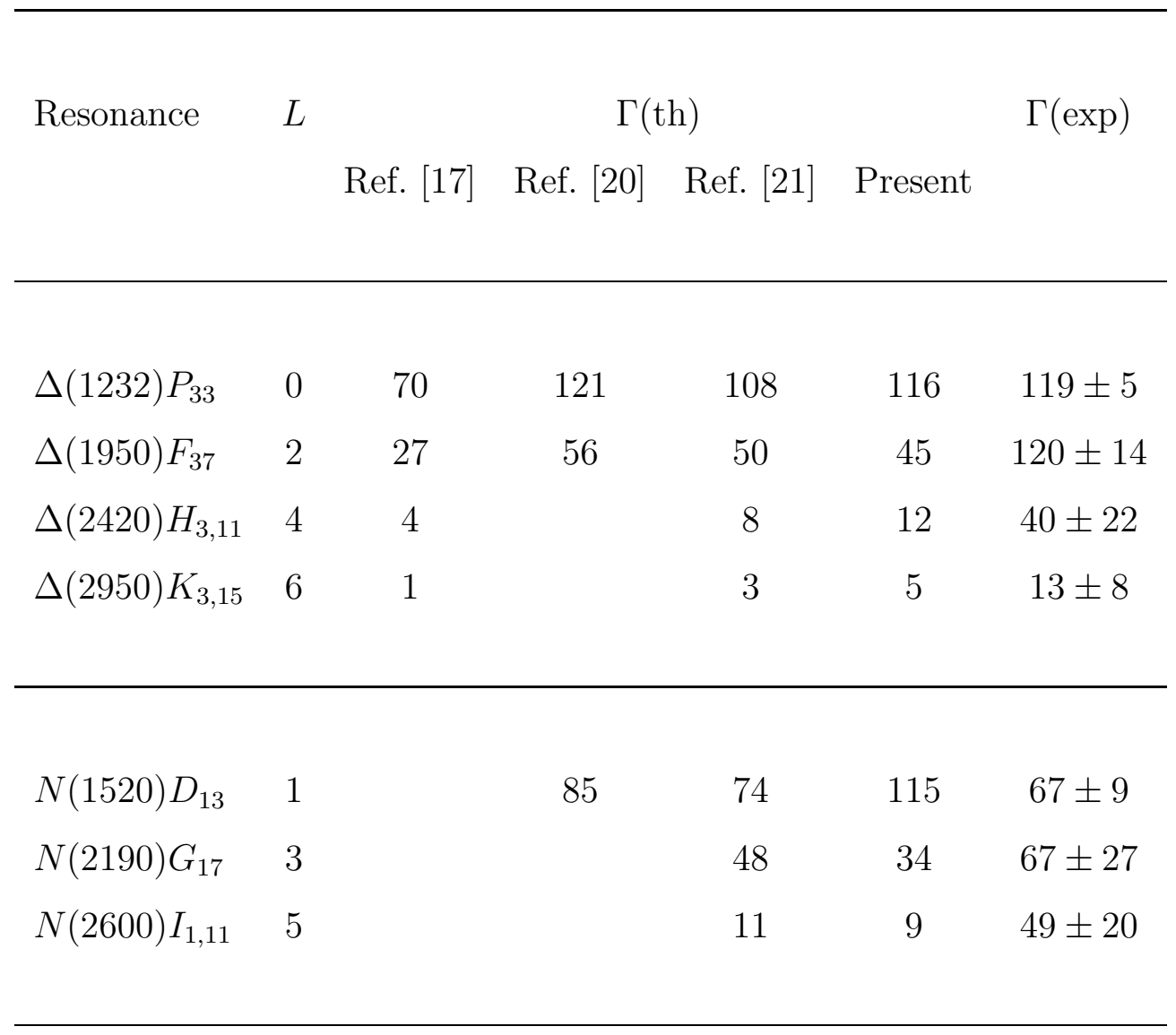

